\title{
Metal-Enhanced Fluorescence of Chlorophylls in Single Light-Harvesting Complexes
}

\author{
Sebastian Mackowski, ${ }^{\dagger}$ Stephan Wörmke, ${ }^{\dagger}$ Andreas J. Maier, ${ }^{\dagger}$ \\ Tatas H. P. Brotosudarmo, ${ }^{\ddagger}$ Hayk Harutyunyan, ${ }^{\dagger}$ Achim Hartschuh, ${ }^{\dagger}$ \\ Alexander 0. Govorov, ${ }^{\S}$ Hugo Scheer, ${ }^{\ddagger}$ and Christoph Brăuchle ${ }^{\star, \dagger}$ \\ Department of Chemistry and Biochemistry and Center for Nanoscience, \\ Ludwig-Maximilian-University, Butenandtstrasse 11, D-81377 Munich, Germany, \\ Department of Biology I-Botany, Ludwig-Maximilian-University, \\ D-80638 Munich, Germany, and Department of Physics and Astronomy, \\ Ohio University, Athens, Ohio 45701
}

Received November 2, 2007; Revised Manuscript Received December 10, 2007

\begin{abstract}
Ensemble and single-molecule spectroscopy demonstrates that both emission and absorption of peridinin-chlorophyll-protein photosynthetic antennae can be largely enhanced through plasmonic interactions. We find up to 18-fold increase of the chlorophyll fluorescence for complexes placed near a silver metal layer. This enhancement, which leaves no measurable effects on the protein structure, is observed when exciting either chlorophyll or carotenoid and is attributed predominantly to an increase of the excitation rate in the antenna. The enhancement mechanism comes from plasmon-induced amplification of electromagnetic fields inside the complex. This result is an important step toward applying plasmonic nanostructures for controlling the optical response of complex biomolecules and improving the design and functioning of artificial light-harvesting systems.
\end{abstract}

Strong enhancement of electromagnetic fields generated through plasmon resonances in metal films and particles has recently stimulated a considerable interest in diverse research fields such as optical spectroscopy, cell imaging, quantum information processing, nanophotonics, and biosensors. ${ }^{1-5}$ This versatility results from a dramatic influence that plasmons impose on the absorption and emission properties of nearby located dipoles, for example, semiconductor nanocrystals and nanowires ${ }^{6-12}$ or dye molecules. ${ }^{13-18}$ Optical response of an emitter coupled to a plasmonic structure depends upon spatial arrangement as well as spectral characteristics of a studied system. Remarkable progress has been made in on-demand design of metal nanostructures, which is essential for tuning the resonance frequency and thus the coupling strength. ${ }^{13,14,19}$ Complementary efforts focused on developing advanced experiments to study dipoles placed in the vicinity of a metal nanoparticle have shed light on the interplay between radiative and nonradiative processes in these systems. ${ }^{16,18}$ This very relation determines whether

\footnotetext{
* Corresponding

cup.uni-muenchen.de.

author.

E-mail:

christoph.braeuchle@

Department of Chemistry and Biochemistry and Center for Nanoscience, Ludwig-Maximilian-University.

$\doteqdot$ Department of Biology I-Botany, Ludwig-Maximilian-University.

$\S$ Ohio University.
}

the fluorescence is enhanced ${ }^{9-11,16}$ or quenched due to the dominating role of nonradiative energy transfer from the dipole to the metal. ${ }^{15,18}$

Metal-enhanced fluorescence (MEF) has been observed for many hybrid systems that include nanocrystals on corrugated metal surfaces, ${ }^{10,11}$ dye molecules coupled to metal nanoparticles, ${ }^{18}$ and nanocrystal-nanoparticle bioconjugates. ${ }^{8}$ In all these cases, very stable and highly fluorescing emitters have been selected. It would be, however, highly desirable to apply MEF to weakly fluorescing systems such as DNA, ${ }^{20}$ carbon nanotubes ${ }^{21}$ or, yet experimentally unexplored in this context, light-harvesting complexes. These latter proteinpigment systems, which contain chlorophyll (Chl) and carotenoid molecules embedded in a protein matrix, participate in the photosynthesis process by collecting sunlight energy and transferring it to reactions centers. The presence of fluorescing Chls and the protein, separated by a few nanometers, renders light-harvesting complexes ideal for studying the protein energy landscape, a timely concept in biophysical chemistry. ${ }^{22}$ Intriguing is also a possibility to use metal nanoparticles for tuning the energy transfer between carotenoids and Chls as well as between Chls themselves, which may help design and optimize artificial light-harvesting structures. ${ }^{23-25}$ 

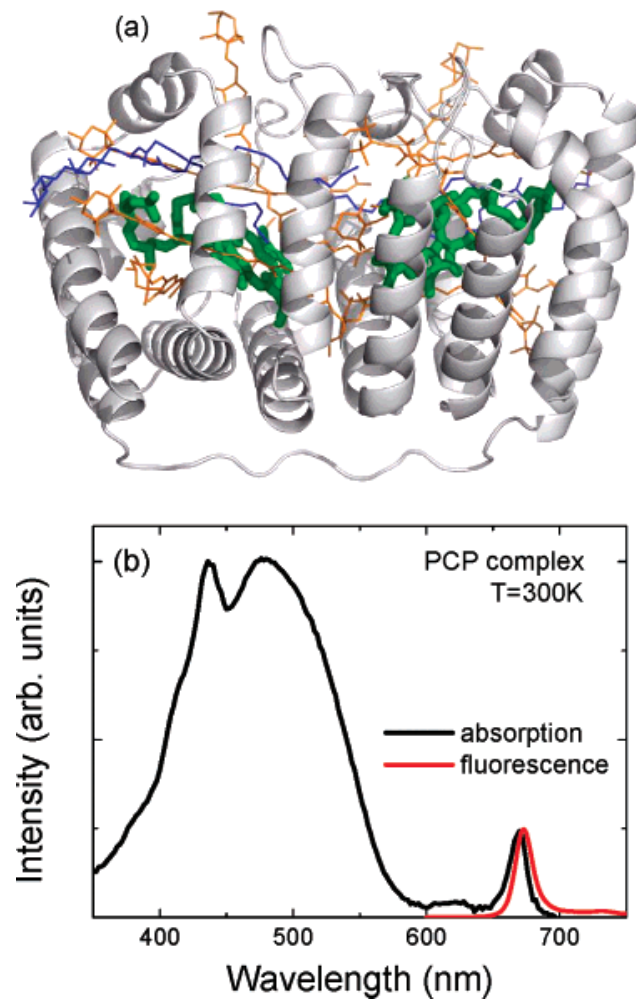

Figure 1. (a) Structure of a PCP monomer: eight peridinins (orange), two chlorophylls (green), and a lipid molecule are embedded in a protein matrix of which only the backbone is shown. PDB entry 1PPR (27). (b) Room-temperature absorption (black) and fluorescence spectra (red) of N-PCP reconstituted with Per and $\mathrm{Chl} a$. Fluorescence was excited at $532 \mathrm{~nm}$.

The structure of a single monomer of the peridininchlorophyll-protein (PCP), a water-soluble peripheral lightharvesting antenna from dinoflagellate Amphidinium carterae, is depicted in Figure 1a. Organized in two clusters, it contains two Chl $a$ (green) and eight peridinin (Pers, orange) pigments that are closely packed within the hydrophobic cavity formed by the protein and a lipid (blue). ${ }^{26}$ The distance between the two Chls $a$ is $17.4 \AA$. The absorption spectrum (Figure 1b) shows that PCP utilizes the carotenoid, Per, as its primary pigment, which is responsible for the dominant absorption band in the blue-green spectral region (350 to $550 \mathrm{~nm})$. The Chls absorb around $668 \mathrm{~nm}\left(\mathrm{Q}_{\mathrm{Y}}\right.$ band) and $440 \mathrm{~nm}$ (Soret band). The Per absorption range corresponds well to plasmon energies of metal nanoparticles. The fluorescence of PCP originates from the $\mathrm{Q}_{\mathrm{Y}}$ transition of $\mathrm{Chl}$ $a$ and it is located at $673 \mathrm{~nm}$ (Figure 1b), more than $100 \mathrm{~nm}$ below the Per absorption band. The overlap between Per absorption and plasmon frequencies together with the large Stokes shift renders PCP an ideal system for studying the impact of plasmonic interactions on the excitation and recombination dynamics in this simple light-harvesting biomolecule.

This proof-of-concept work demonstrates that plasmon excitations in a silver island film (SIF) induce dramatic fluorescence enhancements in individual pigment-protein complexes. Fluorescence spectroscopy of single PCP complexes deposited randomly on SIF reveals an average emission intensity increase by a factor of 6 with some complexes showing even an 18 -fold enhancement. Remarkably, there is no influence of SIF on the emission energy, indicating that the pigment-protein complexes remain intact near the metal surface. Steady-state and time-resolved experiments on ensembles show similar MEF when PCP is excited into Per and Chl $a$ absorption. Considering faster photobleaching of SIF-deposited PCP, the results suggest that the dominant mechanism of MEF is a strong increase of the excitation rate in the light-harvesting complex, which is highly favorable as it improves light collection efficiency. We envision a breakthrough in developing strategies for efficient light-harvesting systems through controlled fabrication of hybrid structures composed of the natural lightharvesting antennae and inorganic systems including metal nanoparticles.

PCP complexes were obtained by biochemical reconstitution of the N-terminal domain apoprotein with Per and Chl $a$, as described in detail previously. ${ }^{27}$ The final product was equilibrated with TRIS buffer $(5 \mathrm{mM}, \mathrm{pH} 7.6)$ and kept frozen until used. Silver island films were prepared by reducing an aqueous silver nitrate solution. ${ }^{10}$ All chemicals were purchased from Sigma-Aldrich and used as received. Briefly, freshly prepared aqueous $\mathrm{NaOH}(1.25 \mathrm{M})$ was added to a silver nitrate solution. The precipitate was redissolved by adding $\mathrm{NH}_{4} \mathrm{OH}$, and the solution was cooled to $\sim 5{ }^{\circ} \mathrm{C}$ under stirring. After adding D-glucose, 12 clean microscope cover slips were dipped in the solution, which was then heated up to $30^{\circ} \mathrm{C}$. The resulting Ag-covered glass coverslips were examined using absorption spectroscopy and atomic force microscopy (AFM). AFM images taken with an MFP3D instrument (Asylum Research) using a "Nanosensors Pointprobe-Plus" cantilever showed metal islands with diameters from 70 to $140 \mathrm{~nm}$ and heights between 30 and $40 \mathrm{~nm}$ (see Figure 1SM of Supporting Information). The resulting plasmon resonance has a maximum at $450 \mathrm{~nm}$ (see Figure 2SM of Supporting Information) and the line width of about $150 \mathrm{~nm}$, and thus matches nicely the PCP absorption.

Desired concentrations of the light-harvesting complexes were achieved by diluting the PCP solution in $2 \%$ aqueous poly(vinyl alcohol) (PVA) solution (Sigma-Aldrich). Subsequently, $20 \mu \mathrm{L}$ drop of the sample was spin-coated on the SIF coverslips. Samples prepared on bare glass coverslips in exactly the same way were used as reference. The thickness of the PVA layer containing PCP complexes was about $100 \mathrm{~nm}$. Steady-state fluorescence spectra of the ensembles and single PCP complexes were measured at room temperature with a modified scanning confocal microscope (Zeiss LSM 410) equipped with a high NA oil-immersion objective (Zeiss Plan-Apochromat 63x/1.4). The samples were excited at 532 and $632 \mathrm{~nm}$. The emission was extracted using band-pass filters (HQ 670/10, Chroma), dispersed by an Amici prism and detected by a Peltier-cooled chargecouple device camera (Princeton Instruments, EEV 1300/ 100-EMB-chip) with exposure times of $1 \mathrm{~s}$. Fluorescence lifetimes were measured with a time-correlated single photon counting technique (Becker \& Hickl, SPC-140 TCSPCBoard). In this case, a 530/10 nm bandpass filter (Thorlabs) was used to select the excitation wavelength from the white 

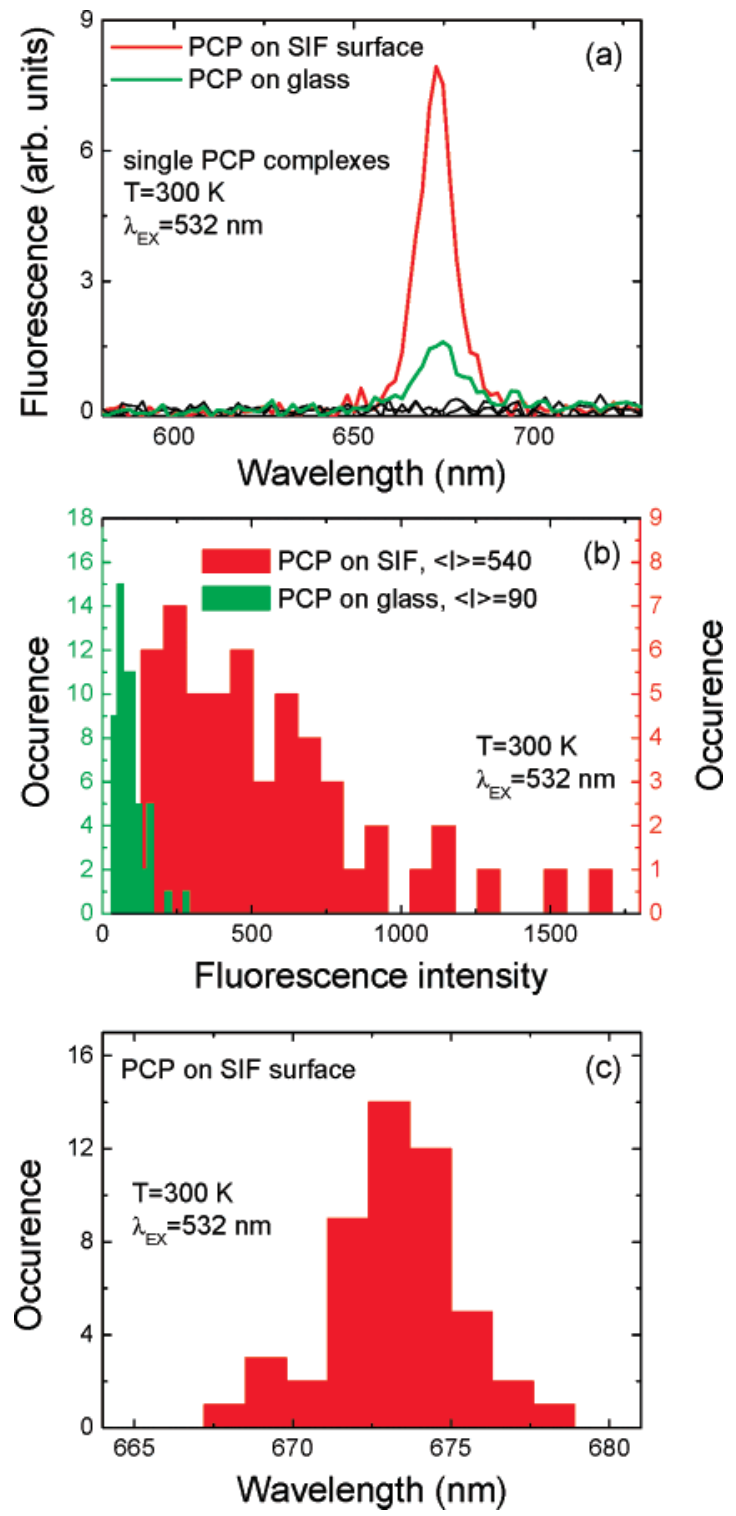

Figure 2. (a) Typical fluorescence spectra of individual PCP complexes on glass (green) and SIF-coated (red) coverslips excited at $532 \mathrm{~nm}$. Black curve corresponds to the background. (b) Distribution of fluorescence intensity measured for over sixty single PCP complexes on glass (green) and SIF-coated coverslips. (c) Distribution of maximum emission energies for PCP complexes deposited on SIF-covered coverslip.

light supercontinuum given by a Ti:Sapphire laser (Coherent Mira, 150 fs pulse width, $75.3 \mathrm{MHz}$ repetition rate). The excitation beam was guided into the microscope (Nikon Eclipse TE2000-S) and focused onto the sample using an oil-immersion objective (Nikon Plan Fluor $\mathrm{S}, \mathrm{NA}=1.3$ ). The sample fluorescence spectrally filtered with a 670/10BP filter (Chroma) was detected with a fast APD (APD-MPD5CTC PicoQuant, full width at half-maximum of $\sim 27 \mathrm{ps}$ ).

Single-molecule spectroscopy provides a very direct insight into the optical properties of nanostructures ${ }^{28}$ by revealing information not only about average values but also intrinsic distributions of measured parameters. Representative spectra, measured with identical laser power of $15 \mu \mathrm{W}$ for individual PCP complexes deposited on SIF (red) and glass (green) coverslips, are shown in Figure 2a. The excitation wavelength of $532 \mathrm{~nm}$ corresponds to the Per absorption (Figure 1b), therefore the Chls are excited via the energy transfer, which randomizes any polarization of the excitation. The emission of the PCP complex on the glass coverslips is comparable to previously reported results..$^{29,30}$ By contrast, the fluorescence intensity of the complex placed close to SIF exhibits a huge, 6-fold enhancement. This is the first experimental demonstration that interaction with plasmons can lead to dramatic MEF of Chls in light-harvesting complexes. In Figure 2b, we compare the intensities measured for over sixty PCP complexes on glass (green) and SIF surfaces (red). On average, the fluorescence increases by a factor of 6 (average intensities for PCP on glass and on SIF are 90 and 540 counts per second, respectively), but for some complexes we observe even an 18 -fold increase. The significant broadening of the intensity distribution obtained for PCP complexes on SIF layer provides not only a clear proof for efficient coupling between metal and biomolecule but is also a direct consequence of inhomogeneities characteristic for the chosen geometry. ${ }^{10}$ First, the size variation of Ag nanoparticles in the SIF layer, as observed using AFM, results in a broad distribution of fluorescence enhancement factors for individual PCP complexes. Therefore, we would expect the PCP fluorescence intensity to reflect the heterogeneity of the system. In addition, the interaction between the metal surface and the pigments (Per and $\mathrm{Chl}$ ) depends on the distance: fluorescence is quenched for PCP complexes located directly on the SIF surface, ${ }^{27}$ while fluorescence of the ones separated by approximately $50 \mathrm{~nm}$ or more should remain unaffected. A signature of the latter is presumably the fraction of PCP complexes on SIF with intensities below 200 counts per second, which is comparable to signals measured for the reference sample. On the other hand, an indirect indication for the quenching could be smaller (by approximately by $15 \%$ ) number of PCP molecules per unit area observed for SIF-covered coverslips.

Importantly, neither the line width nor the wavelength of the PCP fluorescence are affected by the proximity of the silver metal film. The distribution of maximum emission wavelengths measured for over sixty PCP complexes on SIF (Figure 2c) is centered at $673 \mathrm{~nm}$, and thus matches almost perfectly the distributions obtained previously for PCP on the glass surface. ${ }^{30}$ We therefore conclude that the protein surrounding the pigments remains intact during the experiment, and the complex as a whole maintains its lightharvesting function. This is also supported by the efficient energy transfer between Per and Chl (used for Chl excitation), which is critically depending on the distance between the pigments. This observation shows considerable promise for applying plasmonic nanostructures to tune the spectroscopic properties of natural and artificial photosynthetic complexes, including absorption cross-sections, fluorescence dynamics and rates of energy transfer. It requires a further development of ways for better controlling the homogeneity of the fluorescence enhancement of biomolecules, through precise nanofabrication of plasmonic structures and tuning the distance and orientation between metal nanoparticles and light-harvesting complexes. 

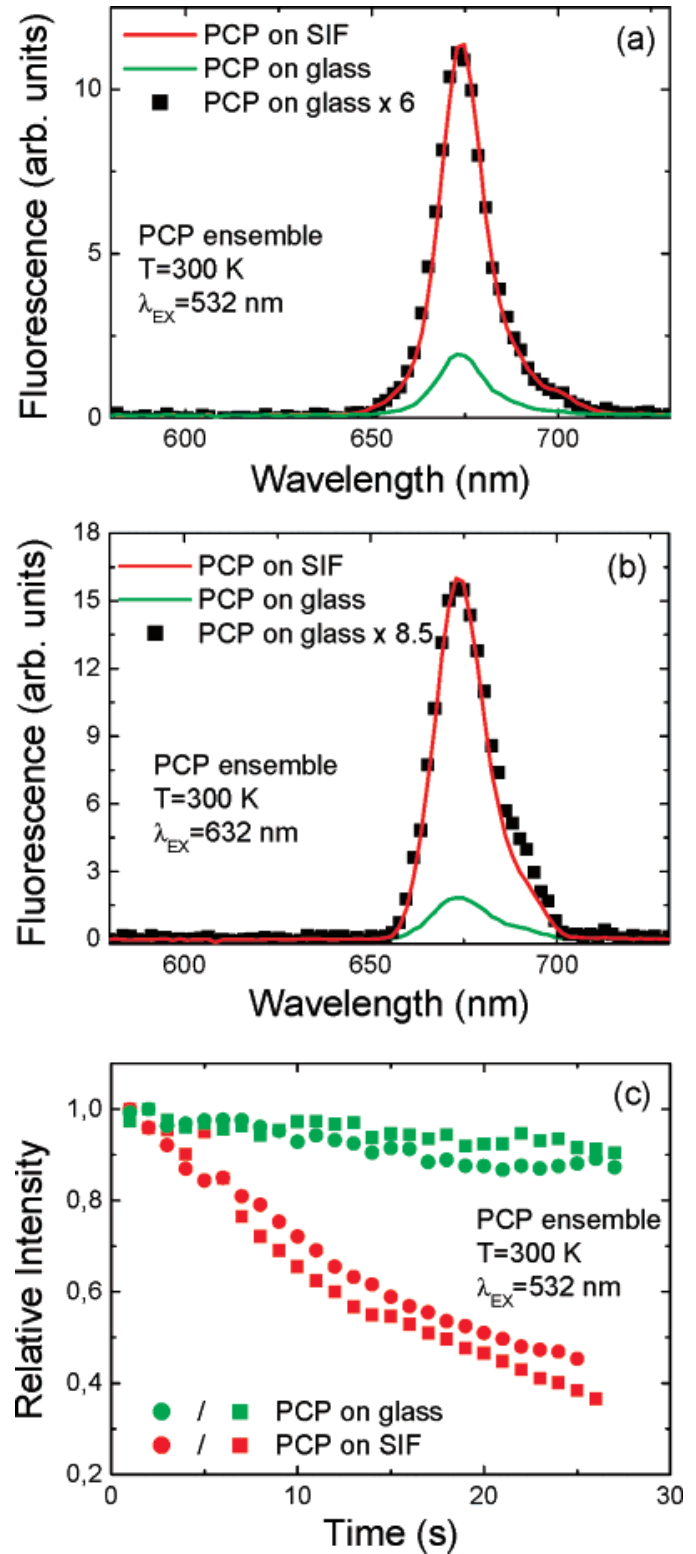

Figure 3. (a) Average fluorescence emission spectra measured for PCP ensembles on bare glass (green) and SIF-coated coverslips (red) excited at $532 \mathrm{~nm}$. Black points correspond to the spectrum measured for PCP on glass multiplied by a factor of six. (b) Average fluorescence emission spectra measured for PCP ensembles on bare glass (green) and SIF-coated coverslips (red) excited at $632 \mathrm{~nm}$. Black points correspond to the spectrum measured for PCP on glass multiplied by a factor of 8.5. (c) Temporal evolution of the fluorescence intensity of PCP ensembles on bare glass (green) and SIF-coated coverslips (red). The excitation wavelength was 532 $\mathrm{nm}$. Dots and squares represent two independent measurements for each configuration.

Ensemble fluorescence was measured on more concentrated PCP samples deposited under otherwise identical conditions on SIF and bare glass coverslips. To probe MEF effect for PCP complexes excited selectively in the Per or Chl absorption bands (Figure 1), the samples were excited at 532 (Figure 3a) and $632 \mathrm{~nm}$ (Figure 3b). In each case, 10 spectra were collected at different locations to judge the influence of local fluctuations of PCP concentration; the preparations were found homogeneous with standard intensity deviations of less than 15\%. The spectra shown in Figure
3 are close to average ones with red and green lines representing PCP complexes deposited on SIF and bare glass coverslips, respectively. For both excitation wavelengths, the fluorescence intensity of PCP complexes on the metal surface is clearly increased compared to the reference samples. For PCP excited into Per absorption (532 nm), we observe a 6-fold enhancement of the fluorescence intensity in perfect agreement with the single molecule results. The enhancement is even larger (8.5-fold), when the light-harvesting complexes are excited directly into the blue wing of the Chl $\mathrm{Q}_{\mathrm{Y}}$-band $(632 \mathrm{~nm})$, although the absorption spectrum of the SIF and the wavelength dependence of the electric-field enhancement (see below) would suggest the reverse. However, the excitation mechanisms for these two wavelengths are qualitatively different (see Figure 3SM of Supporting Information). With the laser wavelength of $632 \mathrm{~nm}$, only Chl molecules are excited and thus the increase of the fluorescence intensity is only due to plasmonic interaction with Chls. On the other hand, the excitation at $532 \mathrm{~nm}$ corresponds almost exclusively to Per absorption and the excited states of the Chls are populated via subsequent energy transfer. ${ }^{30}$ Because the Per absorption as well as the efficiency of energy transfer could be affected by the proximity of the metal film, ${ }^{20}$ both these processes contribute to the experimentally observed fluorescence enhancement. As they cannot be separated from each other, we are not able to directly compare the results obtained for the two excitation wavelengths of 532 and $632 \mathrm{~nm}$. Importantly, the wavelength and the line width of the emission remain unaffected by the support, as observed on a single molecule level, and by the excitation wavelength. The curves obtained by multiplying the spectra measured for the reference samples by the respective enhancement factors (black points in Figure 3a,b), match perfectly the fluorescence spectra of PCP complexes on SIF substrates. This reinforces the conclusion reached from single-molecule data that the protein structure of PCP complexes are unaffected in any significant way by the presence of metal nanostructures in its vicinity.

Another facet of how plasmonic excitations in SIF layer influence the spectroscopic properties of PCP complexes is displayed in Figure 3c, where we plot time evolution of the fluorescence intensity measured for PCP ensembles excited at $532 \mathrm{~nm}$. The emission intensity of the reference sample decreases by $\sim 10 \%$ over the first $30 \mathrm{~s}$; ; that of the PCP complexes coupled to SIF decreases during this time by $\sim 50 \%$. The increased photobleaching on SIF results probably from a larger number of photocycles realized by $\mathrm{Chl}$ molecules in this light-harvesting system. This indicates that the dominant mechanism responsible for the fluorescence enhancement is a dramatic increase of the light absorption. There is also increased photobleaching on SIF surface if PCP excited into the $\mathrm{Chl}$ absorption $(632 \mathrm{~nm})$, but the effect is less pronounced. It should be noted, however, that direct excitation of Chls leads already to a faster photobleaching of the PCP complexes deposited on the glass surface, as compared to excitation via Per. This can be intuitively understood by an incomplete energy transfer from Per to $\mathrm{Chl},{ }^{20}$ resulting in a smaller number of photocycles in a given time. 


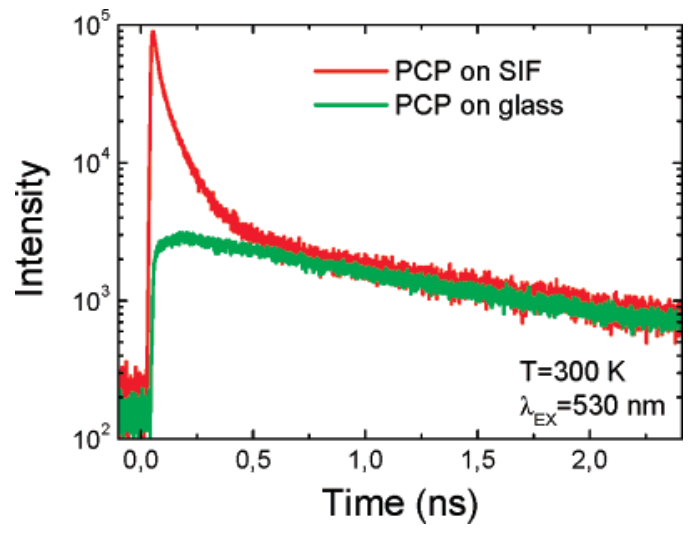

Figure 4. Time-resolved fluorescence of PCP on bare glass (green) and SIF-coated coverslips (red). The excitation wavelength was 530 $\mathrm{nm}$, and the pulse width was $150 \mathrm{fs}$.

Important information about the exact mechanism of fluorescence enhancement and relative contributions of nonradiative and radiative processes can be deduced from the excited-state dynamics. In Figure 4, the fluorescence decay of PCP complexes on SIF (red line) is compared with that of the reference sample (green line). The fluorescence signal of PCP on glass decays monoexponentially with a lifetime of $\tau=3.68 \mathrm{~ns}$. In contrast, the fluorescence of PCP complexes deposited on SIF decays much faster and features multiexponential behavior. The lifetime of the high-intensity component is as short as $200 \mathrm{ps}$. At later times, the trajectory gradually approaches that of $\mathrm{PCP}$ on glass coverslips; at times $>1$ ns both curves are parallel to each other. The complex, multiexponential behavior is another aspect of the aforementioned sample heterogeneity with contributions from PCP complexes interacting with metal nanostructures of different sizes, as well as distribution of the interaction strength due to changes in distances between the SIF layer and the pigments in the complex.

The experimentally observed MEF of single light-harvesting complexes can be qualitatively analyzed with a simple model that has been applied previously to metal nanoparticle (NP) - nanocrystal assemblies. ${ }^{8,10}$ This analysis also provides an intuitive scenario, where an increased absorption in the presence of metal film is the dominant mechanism of fluorescence enhancement. For simplicity, we calculate the plasmonic interaction between a light-harvesting PCP complex and a Ag NP with a diameter of $80 \mathrm{~nm}$ and assume that the fluorescence of PCP is nonpolarized. This neglects the size distribution of the metal nanoparticles in SIF, as well as any polarization and anisotropy effects of PCP emission (i.e., two $\mathrm{Chl}$ molecules are approximated by a single dipole). The emission intensity of a molecule in the vicinity of a metal NP can then be expressed as

$$
\begin{gathered}
I_{\text {emiss }, \text { complex }}=\frac{\gamma_{\text {rad }, 0} I_{\text {abs }, 0} P\left(\lambda_{\text {ex }}\right) P\left(\lambda_{\text {emiss }}\right)}{\gamma_{\text {transfer }}+\gamma_{\text {rad }, 0} P\left(\lambda_{\text {emiss }}\right)+\gamma_{\text {non }- \text { rad }}}= \\
P\left(\lambda_{\text {ex }}\right) P\left(\lambda_{\text {emiss }}\right) \frac{\gamma_{\text {tot }, 0}}{\gamma_{\text {tot }}} I_{\text {emiss }, 0}
\end{gathered}
$$

where $\lambda_{\text {ex }}$ and $\lambda_{\text {emiss }}$ are the excitation and emission wave- lengths, $\gamma_{\text {tot }, 0}=1 / \tau_{\text {lifetime }, 0}, \gamma_{\text {rad, }, 0}$, and $\gamma_{\text {non-rad }}$ are the total, radiative, and nonradiative relaxation rates of a PCP complex in the absence of metal NP, respectively, $I_{\mathrm{abs}, 0}$ is the absorption of an isolated PCP complex, and $\gamma_{\text {transfer }}$ is the Förster energy transfer rate from the PCP complex to the Ag NP. The relaxation rate of the PCP complex in the vicinity of metal NP is given by

$$
\gamma_{\text {tot }}=\gamma_{\text {transfer }}+\gamma_{\text {rad }, 0} P\left(\lambda_{\text {emiss }}\right)+\gamma_{\text {non-rad }}
$$

The parameter $P(\lambda)$ describes the electric-field enhancement factor at the site of the molecule due to the presence of metal it affects both the absorption and emission properties of the complex.

We now estimate the fluorescence enhancement $P\left(\lambda_{\text {ex }}\right) P\left(\lambda_{\text {emiss }}\right)$ for a PCP complex in PVA matrix in the vicinity of a spherical $\mathrm{Ag}$ NP. The first factor $P\left(\lambda_{\text {ex }}\right)$ described the absorption process, and the second is associated with the emission. The enhancement factors $P(\lambda)$ for both absorption and emission should be averaged over all directions

$$
P(\lambda)=\left(P_{x}+P_{y}+P_{z}\right) / 3=1+2 \frac{R_{\mathrm{NP}}^{6}}{d^{6}}\left|\frac{\epsilon_{\mathrm{Ag}}(\lambda)-\epsilon_{0}}{\epsilon_{\mathrm{Ag}}(\lambda)+2 \epsilon_{0}}\right|^{2}
$$

where $R_{\mathrm{NP}}$ and $d$ are the NP radius and the distance between the NP and the PCP complex, respectively, and $d=R_{\mathrm{NP}}+$ $\Delta$ (see inset in Figure 5). The dielectric constants for $\mathrm{Ag}$ $\epsilon_{\mathrm{Ag}}(\lambda)$ are taken from ref 31 , while for the PVA polymer we use $\epsilon_{0}=2$.2. In Figure 5a, we show the electric-field enhancement $P\left(\lambda_{1}\right) P\left(\lambda_{\text {emiss }}\right)$ calculated for a PCP complex placed $4 \mathrm{~nm}$ from the Ag NP with a radius $R_{\mathrm{NP}}=40 \mathrm{~nm}$. The obtained enhancement factors for the absorption and emission are $P\left(\lambda_{\text {ex }}=532 \mathrm{~nm}\right) \sim 6$ and $P\left(\lambda_{\text {emiss }}=673 \mathrm{~nm}\right)$ $\sim 3.4$, respectively. These values indicate that the fluorescence enhancement observed for single PCP molecules on SIF surface is primarily due to increased probability of photon absorption, $P\left(\lambda_{\text {ex }}\right)$, which results from an excitation wavelength that is closer to the plasmon resonance of the Ag NP than the emission wavelength. Of course, the enhancement factor $P\left(\lambda_{\text {ex }}\right) P\left(\lambda_{\text {emiss }}\right)$ depends strongly on the distance between the Ag NP and the PCP complex and on the NP size. Keeping the NP radius constant at $R_{\mathrm{NP}}=40$ $\mathrm{nm}$, effective enhancement factors of $P\left(\lambda_{\text {ex }}=532 \mathrm{~nm}\right) P\left(\lambda_{\text {emiss }}\right.$ $=673 \mathrm{~nm}) \sim 19-2$ are obtained for Ag NP-PCP distances of $\Delta=4-20 \mathrm{~nm}$. On the other hand, for larger NP sizes of $R_{\mathrm{NP}}=100 \mathrm{~nm}$, the total fluorescence increase factor of the PCP complex separated by $4 \mathrm{~nm}$ is larger than 30 . These values agree well with fluorescence enhancements observed experimentally. The fluorescence decay measured for PCP on SIF (see Figure 4) shows strongly shortened lifetime of PCP complexes that are presumably very close to the $\mathrm{Ag}$ surface. Taking an average decay rate of $1 \mathrm{~ns}^{-1}$, we obtain a fluorescence decrease by a factor of $\gamma_{\text {tot }} \gamma_{\text {tot }, 0} \sim 4$. Because the experimentally observed fluorescence is increased by a factor of $I_{\text {emiss,complex }} / I_{\text {emiss }, 0} \sim 6$ (excitation at $532 \mathrm{~nm}$ ), the effective enhancement factor for PCP com- 

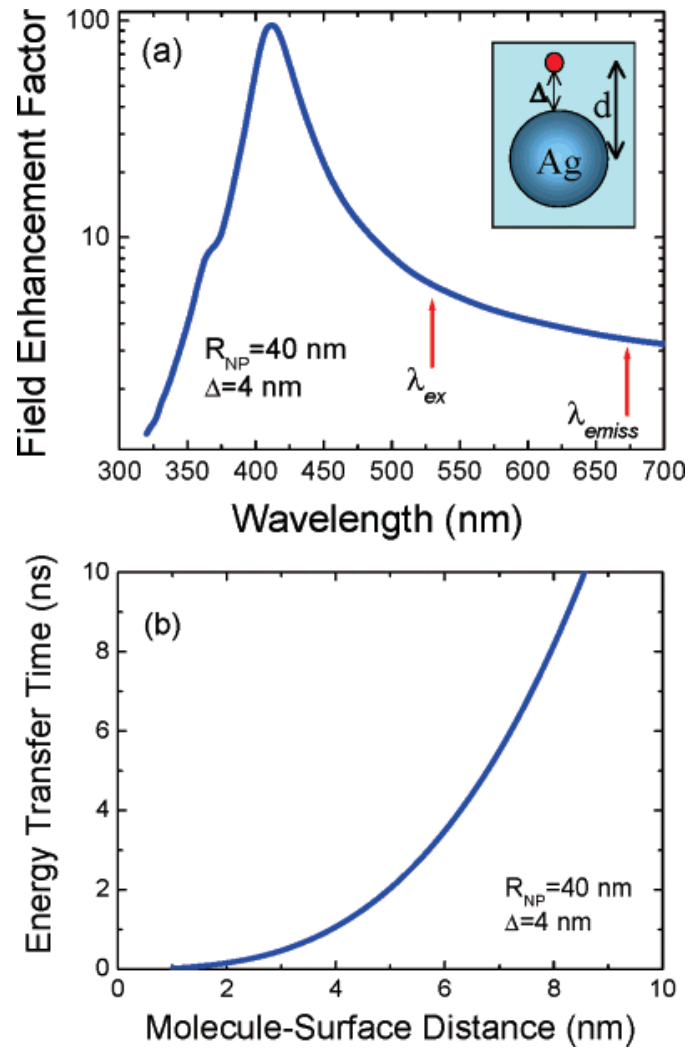

Figure 5. (a) Electric-field enhancement calculated for Ag NP with radius $R=40 \mathrm{~nm}$ and an $\mathrm{Ag} \mathrm{NP}-\mathrm{PCP}$ distance $\Delta=4 \mathrm{~nm}$. The inset shows the geometry used in the calculations. (b) Dependence of the energy transfer time on the distance $\Delta$ between the PCP complex and an $\mathrm{Ag} \mathrm{NP}$ of radius $R=40 \mathrm{~nm}$.

plexes in the vicinity of Ag NPs is remarkably high, reaching $P\left(\lambda_{\text {ex }}\right) P\left(\lambda_{\text {emiss }}\right) \sim 24$.

Strongly shortened lifetimes measured for PCP molecules placed on SIF are also consistent with the picture of Försterlike interactions with metal. Two main mechanisms are responsible for this effect: (a) Energy from a PCP complex is transferred to the metal via Coulomb interaction, creating a short-lived plasmon in an Ag NP. This effect is described by the contribution $\gamma_{\text {transfer }}$ in eq 2. (b) The radiation time becomes shorter due to an enhanced probability to emit a photon from PCP in the vicinity of a metal island. In eq 2, the mechanism (b) is represented by the renormalized emission rate $\gamma_{\text {rad, } 0}=\gamma_{\text {rad, } 0} P\left(\lambda_{\text {emiss }}\right)$. If $R_{\mathrm{NP}} \gg \Delta$, the averaged energy transfer time can be estimated as $\gamma_{\text {transfer }}=\left(\left(\mathrm{ed}_{\mathrm{exc}}\right)^{2}\right) /$ $\left(\hbar \epsilon_{0}^{2}\right)\left(\epsilon_{0}\right) /\left(3 \Delta^{3}\right) \operatorname{Im}\left(\left(\epsilon_{\mathrm{Ag}}-\epsilon_{0}\right)\right) /\left(\epsilon_{0}+\epsilon_{\mathrm{Ag}}\right)$. With an optical dipole moment of PCP $d_{\mathrm{exc}} \approx 1 \AA$ and $\Delta=4 \mathrm{~nm}$, we obtain $\gamma_{\text {transfer }}=1 \mathrm{~ns}^{-1}$ and $\gamma_{\text {tot }}=1.4 \mathrm{~ns}^{-1}$. This corresponds to the lifetime of $\tau_{\text {lifetime }}=1 / \gamma_{\text {tot }}=0.7 \mathrm{~ns}$, which agrees well with the experimental values. The dependence of the energy transfer time on the distance between a PCP complex and the Ag NP plotted in Figure 5b suggests that the complexes exhibiting very short lifetimes must be located within a few nanometers from the metal surface. To obtain the above

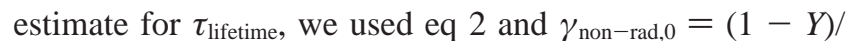
$\tau_{\text {lifetime }, 0}$ and $\gamma_{\text {rad }}=Y \gamma_{\text {rad }, 0} P\left(\lambda_{\text {emiss }}\right)=Y \cdot P\left(\lambda_{\text {emiss }}\right) / \tau_{\text {lifetime }, 0}$, where $Y=0.24$ is the quantum yield of PCP. ${ }^{30}$

In conclusion, we demonstrate that the fluorescence of single light-harvesting PCP complexes deposited on an SIF surface can be enhanced up to 18 -fold due to plasmonic interactions. The pigment-protein complexes remain intact despite the presence of the metal film, but they photobleach more rapidly due to increased number of photocycles. The experimental findings are supported by model calculations of the electric-field enhancement of both absorption and emission, which outweigh any fluorescence quenching due to the Ag NP. Under the experimental conditions, the observed fluorescence enhancement is mainly due to the absorption increase, although it is also possible that the presence of Ag NPs affects the energy transfer from peridinin to chlorophyll. The ability to tune the absorption and emission probabilities of light-harvesting complexes via plasmonic interactions paves a new way for improving the performance of these biomolecules, as well as for designing artificial hybrid light-harvesting systems. Particularly appealing is the prospect of using advanced biochemical techniques to control the morphology of such hybrid structures, thereby optimizing the interactions between biomolecules and metal nanoparticles.

Acknowledgment. The work in Germany was supported by the Deutsche Forschungsgemeinschaft, Bonn (SFB 533, Projects A6 and B7), Nanosystem Initiative Munich (NIM), and Center for Integrated Protein Science Munich (CiPSM). S.M. acknowledges financial support from the Alexander von Humboldt Foundation. The work in the United States was supported by NSF and BioNanoTechnology Initiative at Ohio University.

Supporting Information Available: Atomic force microscopy of SIF surface, absorption spectrum of microscope coverslip with the SIF layer, and excitation and relaxation processes for PCP complex. This material is available free of charge via the Internet at http://pubs.acs.org.

\section{References}

(1) (a) Ozbay, E. Science 2006, 311, 189. Elghanian, R.; Storhoff, J. J.; Mucic, R. C.; Letsinger, R. L.; Mirkin, C. A. Science 1997, 277, 1078.

(2) Lee, J.; Hernandez, P.; Lee, J.; Govorov, A. O.; Kotov, N. A. Nat. Mater. 2007, 6, 291.

(3) Lakowicz, J. R. Plasmonics 2006, 1, 5-33.

(4) Dubertret, B.; Calame, M.; Liebchaber, A. J. Nat. Biotech. 2001, $19,365$.

(5) Slocik, J. M.; Tam, F.; Halas, N. J.; Naik, R. R. Nano Lett. 2007, 7, 1054

(6) Pons, T.; Medintz, I. L.; Sapsford, K. E.; Higashiya, S.; Grimes, A. F.; English, D. S.; Mattoussi, H. Nano Lett. 2007, 7, 3157.

(7) Farahani, J. N.; Pohl, D. W.; Eisler, H. J.; Hecht, B. Phys. Rev. Lett. 2005, 95, 017402 .

(8) Govorov, A. O.; Bryant, G. W.; Zhang, W.; Skeini, T.; Lee, J.; Kotov, N. A.; Slocik, J. M.; Naik, R. R. Nano Lett. 2006, 6, 984.

(9) Kulakovich, O.; Strekal, N.; Yaroshevich, A.; Maskevich, S.; Gaponenko, S.; Nabiev, I.; Woggon, U.; Artmeyev, M. Nano Lett. 2002, 2, 1449.

(10) Ray, K.; Badugu, R.; Lakowicz, J. R. J. Am. Chem. Soc. 2006, 128, 8998.

(11) Shimizu, K. T.; Woo, W. K.; Fisher, B. R.; Eisler, H. J.; Bawendi, M. G. Phys. Rev. Lett. 2002, 89, 117401.

(12) Lee, J.; Javed, T.; Skeini, T.; Govorov, A. O.; Bryant, G. W.; Kotov, N. A. Angew. Chem., Int. Ed. 2006, 45, 4819.

(13) (a) Liu, G. L.; Yin, Y. D.; Kunchakarra, S.; Mukherjee, B.; Gerion, D.; Jett, S. D.; Bear, D. G.; Gray, J. W.; Alivisatos, A. P.; Lee, L. P.; Chen, F. Q. F. Nature Nanotechnol. 2006, 1, 47. (b) Chen, Y.; Munechika, K.; Ginger, D. S. Nano Lett. 2007, 7, 690. 
(14) Muskens, O. L.; Giannini, V.; Sanchez-Gil, J. A.; Rivas, J. G. Nano Lett. 2007, 7, 2871.

(15) Dulkeith, E.; Ringler, M.; Klar, T. A.; Feldmann, J.; Munoz Javier, A.; Parak, W. J. Nano Lett. 2005, 5, 585.

(16) Kühn, S.; Hakanson, U.; Rogobete, L.; Sandoghdar, V. Phys. Rev. Lett. 2006, 97, 017402.

(17) Gerber, S.; Reil, F.; Hohenester, U.; Schlagenhaufen, T.; Krenn, J. R.; Leitner, A. Phys. Rev. B 2007, 75, 073404.

(18) Anger, P.; Bharadwaj, P.; Novotny, L. Phys. Rev. Lett. 2006, 96, 113002.

(19) Haynes, C. L.; Van Duyne, R. P. Nano Lett. 2003, 3, 939.

(20) Lakowicz, J. R.; Shen, Y.; D’Auria, S.; Malicka, J.; Fang, J.; Gryczynski, Z.; Gryczynski, I. Anal. Biochem. 2002, 301, 261.

(21) Hartschuh, A.; Pedrosa, H. N.; Novotny, L.; Krauss, T. D. Science 2003, 301, 1354.

(22) (a) Hofmann, C.; Aartsma, T. J.; Michel, H.; Köhler, J. Proc. Natl. Acad. Sci. U.S.A. 2003, 100, 15534. (b) Wörmke, S.; Mackowski, S.; Brotosudarmo, T. H. P.; Garcia, A.; Braun, P.; Scheer, H.; Hofmann, E.; Bräuchle, C. Appl. Phys. Lett. 2007, 90, 193901.

(23) Berera, R.; Herrero, C.; van Stokkum, I. H. M.; Vengris, M.; Kodis, G.; Palacios, R. E.; van Amerongen, H.; van Grondelle, R.; Gust, D.; Moore, T. A.; Moore, A. L.; Kennis, J. T. M. Proc. Natl. Acad. Sci. U.S.A. 2006, 103, 5343.
(24) (a) Balaban, T. S. Acc. Chem. Res. 2005, 38, 612. (b) Röger, C.; Müller, M. G.; Lysetska, M.; Miloslavina, Y.; Holzwarth, A. R.; Würthner, F. J. Am. Chem. Soc. 2006, 128, 6542.

(25) Polivka, T.; Pellnor, M.; Melo, E.; Pascher, T.; Sundstrom, V.; Osuka, A.; Naqvi, K. R. J. Phys. Chem. C 2007, 111, 467.

(26) Hofmann, E.; Wrench, P. M.; Sharples, F. P.; Hiller, R. G.; Welte, W.; Diederichs, K. Science 1996, 272, 1788.

(27) Brotosudarmo, T. H. P.; Hofmann, E.; Hiller, R. G.; Wörmke, S.; Mackowski, S.; Zumbusch, A.; Bräuchle, C.; Scheer, H. FEBS Lett. 2006, 580,5257 .

(28) Single Molecule Spectroscopy-Nobel Conference Lectures; Rigler, R., Orrit, M., Basché, Th., Eds.; Springer: Berlin, 2001.

(29) Wörmke, S.; Mackowski, S.; Brotosudarmo, T. H. P.; Jung, C.; Zumbusch, A.; Ehrl, M.; Scheer, H.; Hofmann, E.; Hiller, R.; Bräuchle, C. Biochim. Biophys. Acta 2007, 1767, 956.

(30) Mackowski, S.; Wörmke, S.; Brotosudarmo, T. H. P.; Jung, C.; Hiller, R. G.; Scheer, H.; Bräuchle, C. Biophys. J. 2007, 93, 3249.

(31) Palik, E. D. Handbook of Optical Constants of Solids; Academic Press: New York, 1985.

NL072854O 